\title{
Gabor Descriptor for Representation of Spatial Feature
}

\section{Comparison Between the Proposed Descriptor and the Conventional Fourier Descriptor}

\author{
Kohei Arai \\ Information Science Department \\ Saga University, Saga \\ Japan
}

\begin{abstract}
New spatial feature descriptor based on Gabor wavelet function is proposed. The proposed method is compared to Fourier descriptor. The experimental results with Advanced Earth Observing Satellite: ADEOS / Advanced Visible and NearInfrared Radiometer: AVNIR image show an effectiveness of the proposed method. It is found that the restored image quality, in terms of root mean square error between the original and the restored images depends on the support length of the mother wavelet and is much better than that with the conventional Fourier descriptor method for spatial feature description.
\end{abstract}

Keywords-Spatial feature; Gabor wavelet descriptor; Fourier descriptor; wavelet transformation; ADEOS (advanced earth observing satellite); AVNIR (advanced visible and near-infrared radiometer)

\section{INTRODUCTION}

There are various examples of applying wavelet analysis to the processing analysis of earth observation satellite images. A method of superimposing a plurality of visible images after wavelet transform [1], a method of superimposing a plurality of SAR: Synthetic Aperture Radar images with different offnadir angles after wavelet transform [2], and an annual fluctuation pattern of sea surface temperature estimated from satellite data. Method [3] that applies wavelet transform to extract its features, method [4] that applies wavelet transform to the extraction of surface roughness of sea ice, method [5] that extracts spatial features from images extracted from soil moisture, etc. There are [6], [7]. As a report on image processing analysis using the Gabor transform, a report on compression [8] - [11], a report on texture analysis [12] [15], a report on reconstruction [16] - [21], and a report on feature extraction [22] - [25], there are reports on classification [26] - [28].

However, as far as the authors are aware, there is no example of applying wavelet analysis to the method of describing the geometrical features of an image. The Fourier descriptor is well known as a method of describing the geometrical features of an image [29] - [31]. The Fourier descriptor is the one that the contour line (closed curve) extracted from the image is Fourier expanded, and the closed curve is described by a finite number of Fourier expansion coefficients. The Fourier descriptor does not describe the characteristic points of the closed curve in detail but describes all points using the sin function (cos function). Also, some proposals have already been made for the method of applying the Fourier descriptor to open curves.

On the other hand, the Gabor descriptor proposed in this paper takes advantage of the characteristics of Gabor transformation and is devised so that it is possible to describe the complicated part of the contour line in detail. The author considered that the Gabor descriptor enables the description of geometrical features with less information than the Fourier descriptor with less deterioration [32] - [34]. Furthermore, remote sensing satellite image database system allowing image portion retrievals utilizing principal component which consists spectral and spatial features extracted from imagery data is proposed recently [35].

In this paper, the author tries to extend the descriptor by Fourier expansion and opening to the descriptor by Gabor expansion. The proposed method was applied to the Visible and Near Infrared Radiometer: VNIR data of the Advanced Spaceborne Thermal Emission and Reflection Radiometer: ASTER sensor mounted on the Terra satellite to confirm that it is superior to the existing method and to change the support length in Gabor conversion. The author also confirmed the effect of the proposed method.

The next section describes theoretical background and the proposed method for spatial feature descriptor based on Gabor wavelet function. Then some experiments are described followed by conclusion together with some discussions.

\section{Theoretical BACKGRound AND PROPOSED Method}

\section{A. Fourier Expansion and Gabor Wavelet Expansion}

The Fourier transform of the complex function f given the contour line (closed curve etc.) extracted from the image is defined by the equation (1).

$F(\omega)=\int_{-\infty}^{\infty} \overline{e^{l \omega t}} f(t) d t$

The Gabor transform is defined by equations (2) and (3).

$$
\begin{aligned}
& \left.W(\omega, \sigma, k)=\int_{-\infty}^{\infty} \overline{\exp \left(-\frac{(t-k)^{2}}{\sigma^{2}}\right) e^{\imath \omega t}}\right) f(t) d t \\
& =\int_{-\infty}^{\infty} \overline{e^{\imath \omega t}} \exp \left(-\frac{(t-k)^{2}}{\sigma^{2}} f(t) d t\right.
\end{aligned}
$$


However, $\overline{e^{\imath \omega t}}$ represents the complex conjugate of $\mathrm{e}^{\mathrm{i} \omega \mathrm{t}}$ and $\exp \left(-\frac{(t-k)^{2}}{\sigma^{2}}\right) e^{i \omega t}$ expresses the complex conjugate of $\exp \left(-\frac{(t-k)^{2}}{\sigma^{2}}\right) e^{i \omega t} \cdot \omega$ is a parameter in the frequency axis direction, and $\sigma$ and $\mathrm{k}$ are parameters in the time axis direction.

The Gabor transform is a Gaussian function with a window function introduced into the Fourier transform. That is, the Gabor transform is a kind of Fourier transform with window function. In particular, when $k=0$, equation (2) becomes equation (4).

$\left.W(\omega, \sigma, 0)=\int_{-\infty}^{\infty} \overline{\exp \left(-\frac{(t)^{2}}{\sigma^{2}}\right) e^{l \omega t}}\right) f(t) d t$

When $\sigma=\infty$, equation (2) becomes equation (5).

$\left.W(\omega, \infty, k)=\int_{-\infty}^{\infty} \overline{e^{\imath \omega t}}\right) f(t) d t$

Therefore, it can be seen that it does not depend on the parameter $k$.

According to Eq. (2), if the value of the parameter $\sigma$ is large, the influence of the parameter $k$ on $W(\omega, \sigma, k)$ is small. If the value of the parameter $\sigma$ is small, the influence of the parameter $k$ on $W(\omega, \sigma, k)$ is large.

The Gabor transform is not restored to the original data when the inverse transform is performed because it is not an orthogonal transform. However, even in the Fourier transform which is the orthogonal transform, for example, when the inverse transform is performed through the low-pass filter, the original data is not restored. The reason why Gabor transform is not orthogonal transform is that it introduces window function into Fourier transform. In other words, it is necessary to consider the effect of the window function when performing Gabor transformation.

\section{B. Fourier Descriptor and Gabor Wavelet Descriptor}

In the Fourier descriptor, the extracted spatial feature information is Fourier expanded and the expansion coefficient is used as the descriptor. In the Gabor descriptor, the extracted spatial feature information is Gabor expanded, and the expansion coefficient is used as the descriptor. The Fourier descriptor requires operations from $-\infty$ to $\infty$ according to Eq. (1). The Gabor descriptor proposed in this paper does not require operations from $-\infty$ to $\infty$ by introducing the window function (Gaussian function) in Eq. (2). However, it is assumed that the tail of the Gaussian distribution is regarded as zero. From the viewpoint of the above calculation amount, the author thinks that the Vega ball descriptor is superior to the Fourier descriptor.

The number of non-zero Gabor expansion coefficients is called the support length. It can be seen from Eq. (2) that the support length depends on the parameter $\sigma$.

\section{Gabor Descriptor for Closed Curve}

Let $Z(l)=(x(l), y(l))$ be the closed curve. $Z(l)$ is a point that has advanced a distance $l$ on the closed curve from a certain start point on the closed curve as shown in Fig. 1.

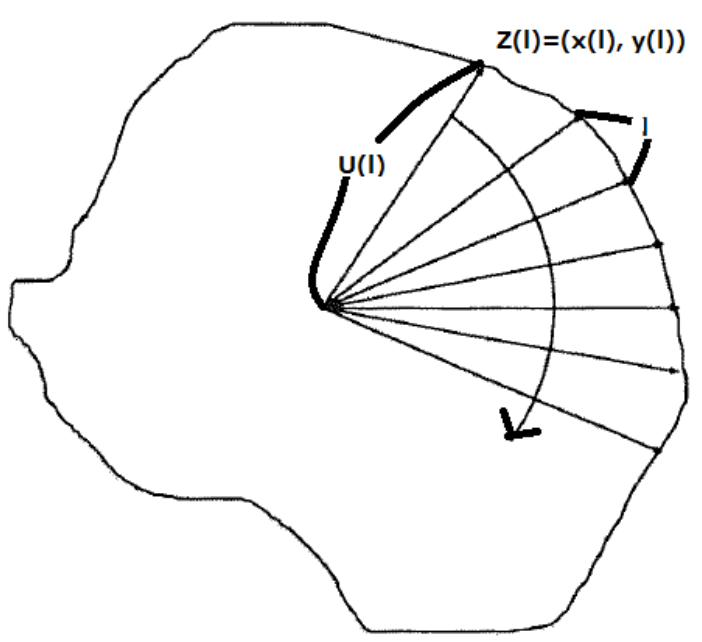

Fig. 1. Concept of the Proposed Wavelet Descriptor.

Here, if the complex function $u(l)$ is defined as follows,

$u(l)=x(l)+i y(l),(i=\sqrt{-1})$

The complex function $u(l)$ is the total length $L$ of the closed curve which is a periodic function. The Gabor descriptor is realized by performing Gabor expansion on the complex function $u(l)$.

\section{Feature of the Proposed Gabor Wavelet Descriptor}

The proposed method considers the information of the edge of the closed curve of the image as a periodic complex function and describes and saves the geometrical feature of the image using the information of the position of a certain starting point on the closed curve and the Gabor expansion coefficient. The proposed method has the following features,

1) When compared with the method of saving edge information as a binary image (method of saving as "face" information), it is superior in terms of the amount of saved data. That is, information of pixels that are not edges is not included.

2) The Fourier descriptor requires operations from $-\infty$ to $\infty$, but the Gabor descriptor does not require operations from $\infty$ to $\infty$.

\section{EXPERIMENTS}

\section{A. Experimental Method}

The data used this time are actual observation images near Mt. Usu acquired by the sensor AVNIR onboard the ADEOS satellite. Fig. 2 shows the aerial photo of Aza-Nakanoshima island near the Mt. Usu while Fig. 3 shows the acquired Advanced Visible and Near Infrared Radiometer: AVNIR (onboard on Terra Satellite) image.

Fig. 3(a) shows an actual observation image, and Fig. 3(b) shows an image resulting from contour extraction. In this experiment, the experiment is performed using Fig. 3(b). Fig. 4 shows the distance from a certain point inside the closed curve in Fig. 3(b) to a point on the closed curve. The purpose of this experiment is to compare the effectiveness of the wavelet descriptor with that of the Fourier descriptor and to 
confirm it by evaluating the restoration accuracy. Therefore, the result obtained by halving the number of Fourier expansion coefficients by a low pass filter is used in the Fourier descriptor, and the result obtained by only the low frequency component is used in the wavelet descriptor. At the same time, the impact of the support length of the wavelet will be examined. In addition, equation (7) is used to evaluate the restoration accuracy.

$J_{1}=\sqrt{\frac{\sum_{s=1}^{N}\left\{(\widehat{x(s)}-x(s)\}^{2}+\left\{(\widehat{y(s)}-y(s)\}^{2}\right.\right.}{N}}$

Note that from Fig. 4, N=256.

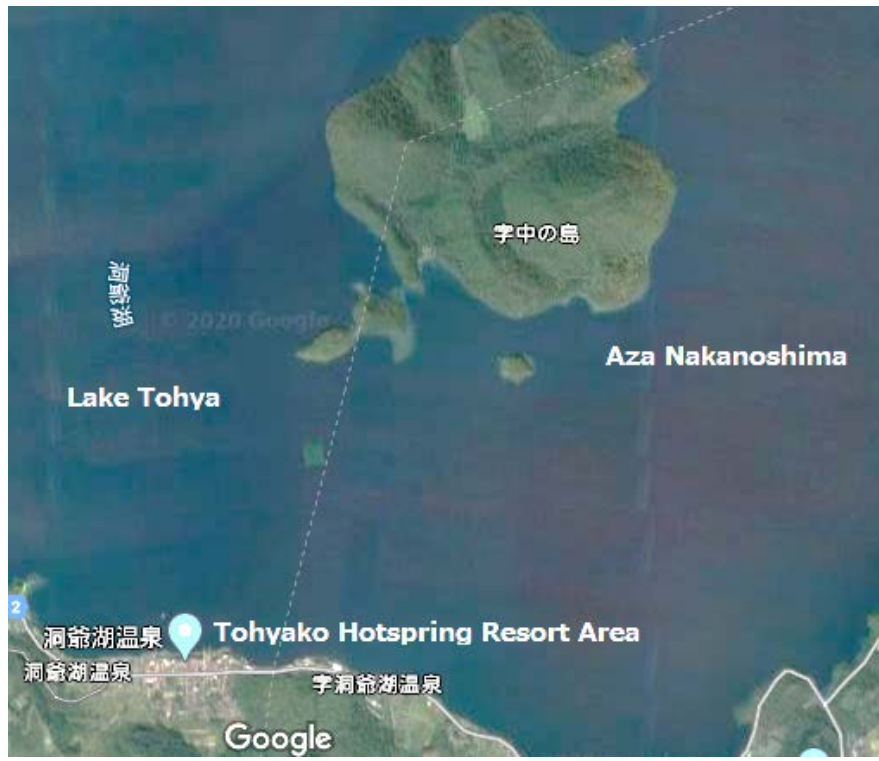

Fig. 2. Google Map (Aerial Photo Image) of Aza Nakanoshima near the Mt.Usu of Intensive Study Area.
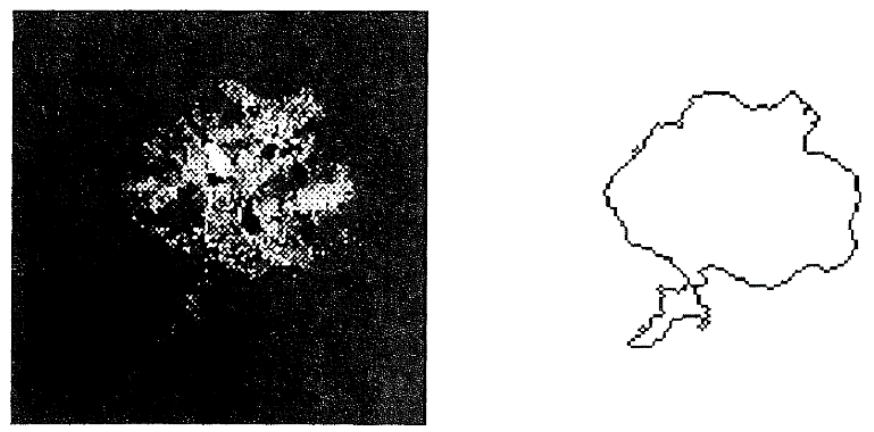

(a) Original AVNIR Image.

(b) Detected Edge.

Fig. 3. Acquired AVNIR Image of Aza-Nakanoshima Island near the Mt. Usu and the Detected Edge Image.

Fig. 4 is an example of constructing a complex function $u(l)$ based on Fig. 3(b). The vertical axis in Fig. 4 is the time axis. The result of projecting the complex function $u(l)$ on the two-dimensional plane orthogonal to the time axis agrees with Fig. 3(b). Since the complex function $u(l)$ is composed of a closed curve, Fig. 4 shows one cycle of the complex function $u(l)$.

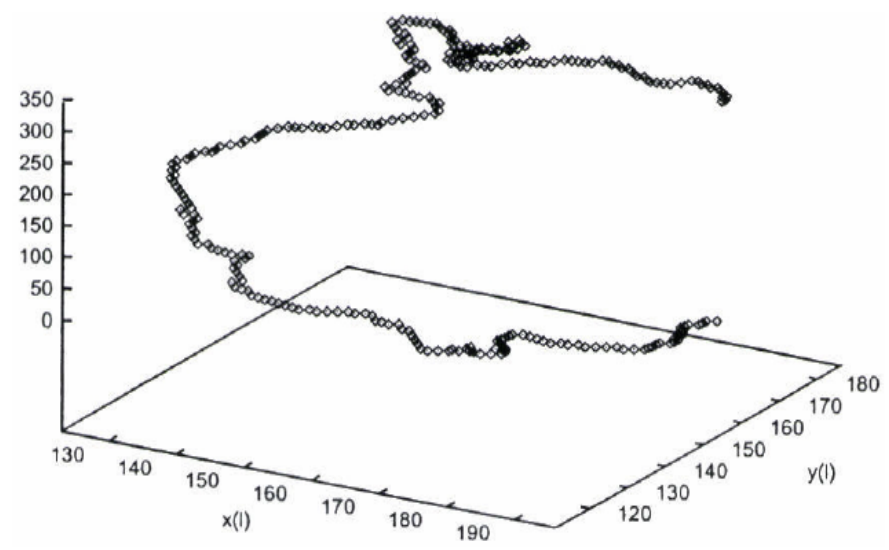

Fig. 4. The Complex Function u(l).

\section{B. Experimental Result}

Fig. 5 shows the real and imaginary parts of Fourier spectrum and the power spectrum when the Fourier expansion is applied to the complex function $u(l)$. Fig. 6 corresponds to the result of the Gabor expansion coefficient when $\sigma=5-40$. Fig. 7 shows the number of non-zero Gabor expansion coefficients (support length) when the parameter $\sigma$ is changed. Fig. 8 shows the restoration accuracy $J_{1}$ when the parameter $\sigma$ is changed. Fig. 6 shows an example of a restored image when the parameters $\sigma=10$ and 20. Table I shows the restoration accuracy $J_{1}$ when the parameter $\sigma$ is fixed and the Gabor expansion coefficient with a large parameter $\omega$ is forcibly set to zero (the restoration accuracy $J_{1}$ is shown when the number of coefficients forcibly set to zero is changed.

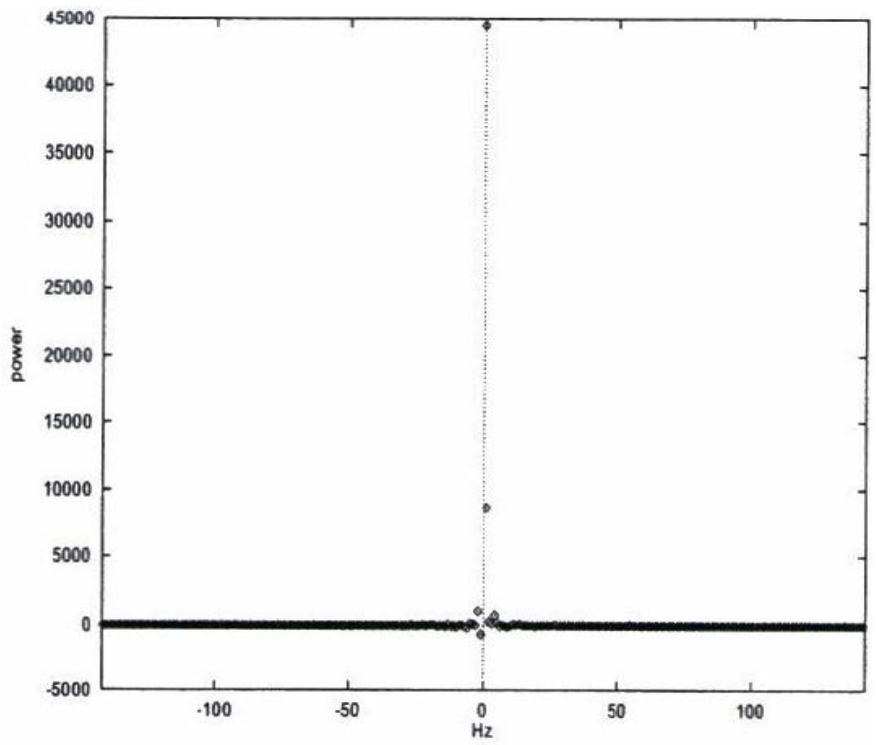

(a) Real Part. 


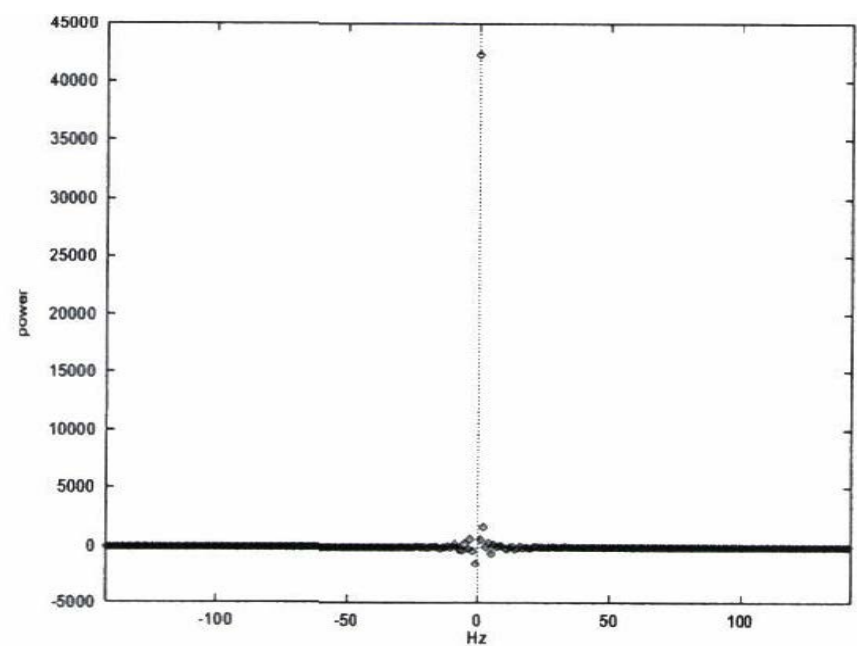

(b) Imaginary Part.

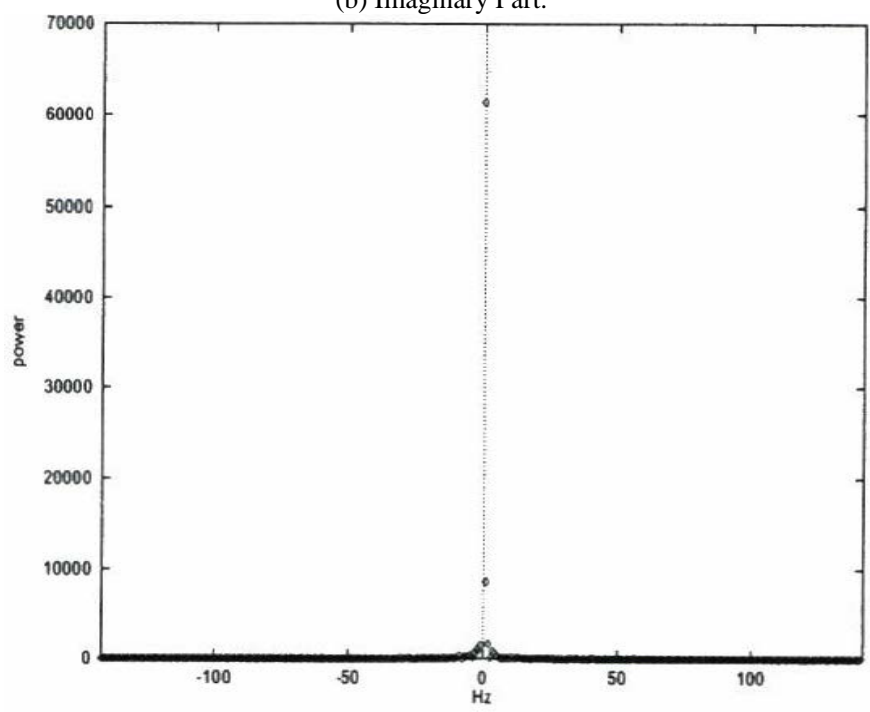

(c) Power Spectrum.

Fig. 5. Real and Imaginary Parts of the Fourier as well as Power Spectrum of $u(l)$.

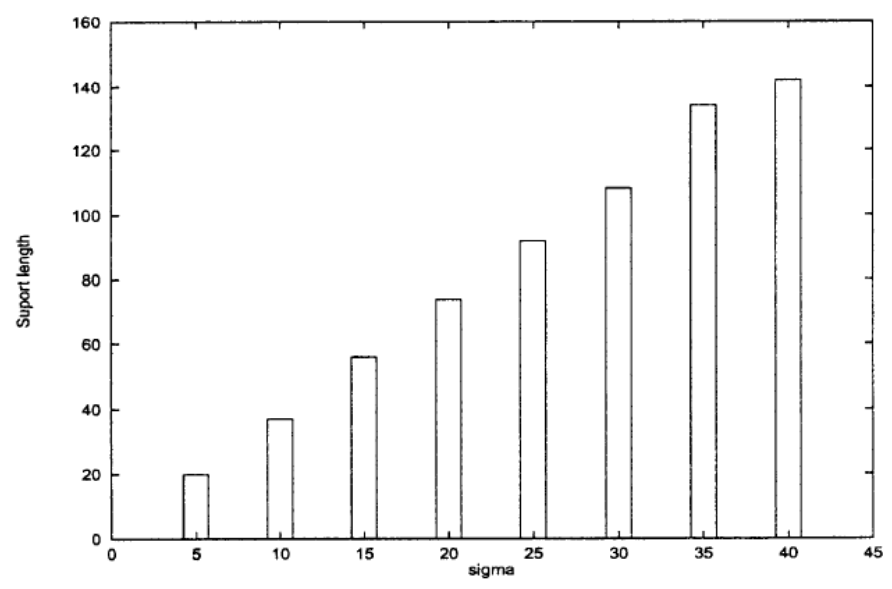

Fig. 6. Support Length for the Designated Parameter $\sigma$.



Fig. 7. Error $\mathrm{J}_{1}$ for the Designated Parameter $\sigma$.

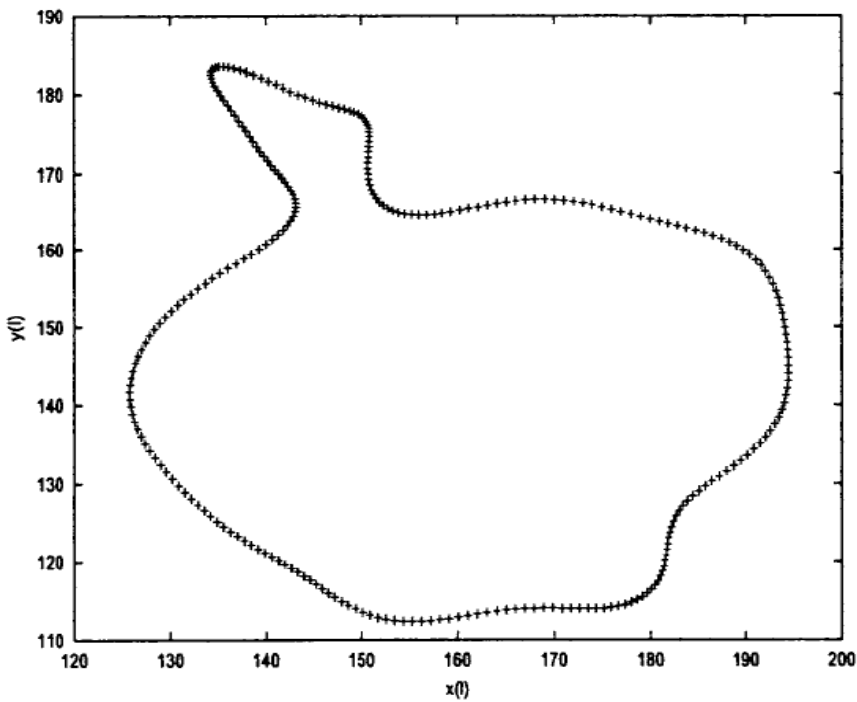

(a) Restored Image $(\sigma=10)$.

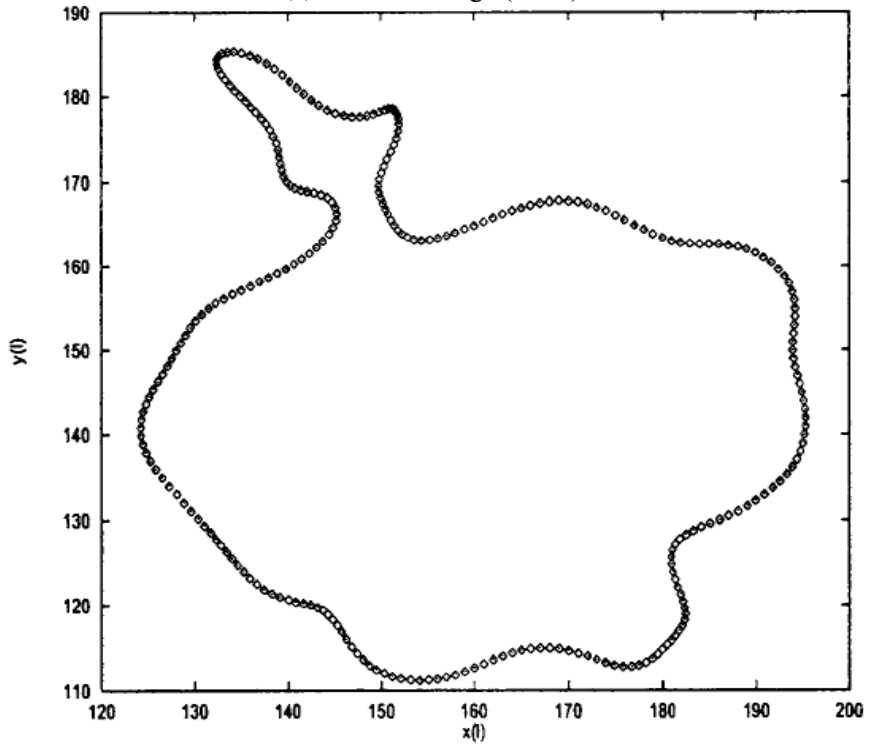

(b) Restored Image $(\sigma=20)$. 




(c) True Contour (the Detected Edge).

Fig. 8. Example of Restored Image for $\sigma=10,20$.

TABLE I. RESTORATION ACCURACY J1 BY THE NUMBER $\Gamma$ OF COEFFICIENTS

\begin{tabular}{|l|l|l|l|l|l|}
\hline$\sigma \mid \gamma$ & 20 & 40 & 60 & 100 & 160 \\
\hline 10 & 1.374 & 1.267 & 1.266 & 1.266 & 1.266 \\
\hline 20 & 1.08 & 0.656 & 0.622 & 0.618 & 0.618 \\
\hline 30 & 1.056 & 0.531 & 0.453 & 0.427 & 0.424 \\
\hline 40 & 1.051 & 0.5 & 0.401 & 0.348 & 0.335 \\
\hline 50 & 1.05 & 0.491 & 0.382 & 0.312 & 0.282 \\
\hline 80 & 1.05 & 0.485 & 0.369 & 0.28 & 0.216 \\
\hline 100 & 1.049 & 0.484 & 0.368 & 0.276 & 0.195 \\
\hline 150 & 1.049 & 0.483 & 0.367 & 0.273 & 0.183 \\
\hline
\end{tabular}

That is, the accuracy $J_{1}$ is shown which is obtained by applying the low pass filter to the result of the Gabor transform of the parameter $\sigma$ and restoring it. Table I shows the restoration accuracy $J_{1}$ by the number $\gamma$ of coefficients that are not forced to zero. The parameter $\gamma$ is a parameter in the frequency axis direction for the low-pass filter, and the parameter $\sigma$ is a parameter in the time axis direction.

\section{Remarks}

As for the relationship between the restoration accuracy and the parameter $\sigma$, it can be seen from Fig. 4 that the support length depends on the parameter $\sigma$. That is, the support length increases as the parameter $\sigma$ increases. From Fig. 7, the restoration accuracy $J_{1}$ depends on the parameter $\sigma$. That is, the restoration accuracy $J_{1}$ improves as the parameter $\sigma$ increases. From Fig. 8, the restoration accuracy of the restored image depends on the support length.

On the other hand, regarding the relationship between the restoration accuracy and the parameter $\omega$, from Table I, the restoration accuracy $J_{1}$, when the number of coefficients that force the Gabor expansion coefficient with a large parameter $\omega$ in each parameter $\sigma$ to zero, is changed is zero. The smaller the number of coefficients to be set, the better.

\section{CONCLUSION}

New spatial feature descriptor based on Gabor wavelet function is proposed. The proposed method is compared to Fourier descriptor. The experimental results with an ADEOS (Advanced Earth Observing Satellite) / AVNIR (Advanced Visible and Near-Infrared Radiometer) image show an effectiveness of the proposed method.

Through experiments with actual remote sensing satellite imagery data, it is found that the proposed description of geometric features of images based on the wavelet transform based on the Daubechies basis is more reproducible than that by existing Fourier descriptors under the condition of the same bandwidth. This is because, when the image space domain is transformed into the Fourier frequency domain and the wavelet frequency domain by the Fourier transform and the wavelet transform, respectively, if the band is halved to the maximum image frequency, the high frequency component disappears in the former case.

On the other hand, in the latter case, it can be said that the high frequency reproducibility is excellent because the high frequency component is preserved even when the latter is divided into the low frequency component. Moreover, it was found that this reproducibility depends on the support length of the basis function in the case of the wavelet descriptor, and when the support length is 2, it exceeds the Fourier descriptor in all cases of 4 or more. From the above, it can be said that the reproducibility of the geometrical description of the image by the wavelet descriptor under the same band limitation exceeds that of the Fourier descriptor.

\section{FUTURE RESEARCH WORK}

Further investigations are required for the validation of the proposed spatial feature extraction method with a variety of imagery data.

\section{ACKNOWLEDGMENT}

The author would like to thank Dr. Kaname Seto (former student of the Saga University) and Mr. Taroh Nakao (former student of the Saga University) for their contributions on this study. The author, also, would like to thank Professor Dr. Hiroshi Okumura and Professor Dr. Osamu Fukuda for their valuable discussions.

\section{REFERENCES}

[1] J.P. Djamdji, A. Bijaoui, and R. Maniere:•gGeo- metrical Registration of Images: The Multiresolution Approach,Photogrammetric Engineering \& Remote Sensing, Vol. 59, No. 5, pp.645-653, (1993).

[2] W.M. Moon, J.S. Won, V. Singhroy, and P.D. Lowman Jr.:ERS-1 and CCRS C-SAR data integration for look-direction bias correction using wavelet transform, Canadian Journal of Remote Sensing, Vol. 20, No. 3, pp.280-285, (1994).

[3] M. Mak:•gOrthogonal Wavelet analysis: Interannual Variability in Sea Surface Tempera - ture, Bulletin of the American Meteorological Society, Vol. 76, No. 11, pp.2179-2186, (1995).

[4] R.W. Lindsay, D.B. Percival, and D.A. Roth rock,:The Discrete Wavelet Transform and the Scale Analysis of the Surface Properties of Sea Ice, IEEE Transactions on Geoscience and Remote Sensing, Vol. 34, No. 3, pp.771-787, (1996).

[5] Z. Hu, Y. Chen, and S. Islam: Multiscaling properties of soil moisture images and decomposi tion of large-and small-scale features using 
wavelet transforms, $\bullet$ hInternational Journal of Remote Sensing, Vol. 19, No. 13, pp.2451-2467,(1998).

[6] Kohei Arai, Kaname Seto, L.M. Jameson: "Extraction of water mass features from satellite image by polar coordinate wavelet, Journal of Visual Information Society, Vol.19, Suppl.No.1, pp.99-102, (1999).

[7] Kohei Arai and Kaname Seto: "Change point extraction of multitemporal earth observation satellite image based on wavelet decomposition and tiling," Journal of Japan Society for Visual Information, Vol.20, Suppl.No.1, pp.285-288, ( 2000).

[8] T. Ebrahimi, and M. Kunt:•gImage compres sion by Gabor expansion,•hOptical engineering, Vol. 30, No. 7, pp.873-880, (1991).

[9] H. Wang and H. Yan:•gAdaptive Gabor discrete cosine transforms for image compression, Electronics letters, Vol. 28, No. 18, pp.1755-1756, (1992).

[10] M.P. Anderson, M.H. Loew, and D.G. Brown: Gabor function-based medical image compres - sion, $\bullet$ Image and vision computing, Vol. 13, No. 7, pp.535-541, (1995).

[11] R.A. Baxter : SAR image compression with the Gabor transform, IEEE Transactions on Geoscience and Remote Sensing, Vol. 37, No. 1, pp.574-588, (1999).

[12] A. Teuner, O. Pichler, and B.J. Hosticka: Unsupervised texture segmentation of images using tuned matched Gabor filters, IEEE Transactions on image processing, Vol. 4, No. 6, pp.863-870, (1995).

[13] D. Dunn, and W.E. Higgins :Optimal Gabor filters for texture segmentation, IEEE Transactions on image processing, Vol. 4, No. 7, pp.947- 964, (1995).

[14] P.P. Raghu, and B. Yegnanarayana: Segmen tation of Gabor-filtered Textures using deter ministic relaxation, IEEE Transactions on image processing, Vol. 5, No. 12, pp.1625-1636, (1996).

[15] R. Porter, and N. Canagarajah: Robust rotation-invariant texture classification: wavelet, Gabor filter and GMRF based schemes, TEE Proc. -Vis. Image Signal Process., Vol. 144, No. 3, pp.180-188, (1997).

[16] J. Yao: Complete Gabor transformation for signal representation, IEEE Transactions on image processing, Vol. 2, No. 2, pp.152-159, (1993).

[17] G. Cristobal, and R. Navarro: Space and frequency variant image enhancement based on a Gabor representation, Pattern recognition letters, Vo1.15. No. 3, pp.273-277, (1994)

[18] T.S. Lee: Image representation using 2D Gabor wavelets, IEEE Transactions on pattern analysis and machine intelligence, Vol. 18, No. 10, pp.959-971, (1996).

[19] R. Navarro, A. Tabernero, and G. Cristobal: Image representation with Gabor wavelets and its applications, $\bullet$ gAdvances in imaging and electron physics, Vol. 97, pp.1-84, (1996).

[20] R. Navarro, A. Tabernero, and G. Cristð®bal: Image representation with Gabor wavelets and its applications, Advances in imaging and elec- tron physics, Vol. 99, pp.329, (1998).

[21] X. Wu, and B. Bhanu: Gabor wavelet representation for 3-D object recognition, IEEE Transactions on image processing, Vol. 6, No. 1, pp.47-64, (1997).

[22] B.J. Super, and A.C. Bovik: Localized mea - surement of image fractal dimension using Gabor filters, •hJournal of visual communication and image representation, Vol. 2, No. 2, pp.114-128, (1991)

[23] J.G. Teti, Jr., and H.N. Kritikos: SAR ocean image decomposition using the Gabor expansion, IEEE Transactions on Geoscience and Remote Sensing, Vol. 30, No. 1, pp.192-196, (1992)
[24] F. Smeraldi, O. Carmona, and J. Bigun: Saccadic search with Gabor features applied to eye detection and real-time head tracking, Image and vision computing, Vol. 18, pp.323-329, (2000).

[25] D.M. Weber, and D.P. Casasent: Quadratic Gabor filters for object detection, IEEE Transactions on image processing, Vo1.10. No. 2, pp.218-230, (2001).

[26] P.P. Raghu, and B. Yegnanarayana: Multispectral image classification using Gabor filters and stochastic relaxation neural network, Neural Networks, Vol. 10, No. 3, pp.561-572, (1997).

[27] L. Wang, C.T. Chen, and W.C. Lin:•gAn ecient algorithm to compute the complete set of discrete Gabor coecients, IEEE Transactions on image processing, Vol. 3, No. 1, pp.87-92, (1994).

[28] J. Yao, P. Krolak, and C. Steele: The generalized Gabor transform, $\bullet h$ IEEE Transactions on image processing, Vol. 4, No. 7, pp.978-988, (1995).

[29] C.T. Zahn and P.Z. Roskies : Fourier descriptors for plane closed curves, IEEE Trans. Computer, Vol. C-21, pp.269-281, (1972).

[30] Takafumi Miyatake, Takashi Matsuyama, Makoto Nagao: "Recognition of curves invariant to affine transformation using Fourier descriptor," Transactions of Information Processing Society of Japan, Vol.24, No.1, pp.64-71, (1983).

[31] Yoshinori Uesaka: "A new Fourier descriptor applicable to open curves," IEICE Transactions, Vo1.J67-A, No.3, pp.166-173, (1984).

[32] Kohei Arai, Kaname Seto and Taro Nakao: "Description of geometrical features of images by wavelet descriptor based on Gabor transform," Proc. of the 30th Annual Meeting of the Remote Sensing Society of Japan, (2001).

[33] Kohei Arai: "Fundamental Theory of Wavelet Analysis" Morikita Publishing, Nov., (2000).

[34] Kohei Arai, Leland Jameson: "How to use earth observation satellite data by wavelet,” Morikita Publishing, July, (2001).

[35] Kohei Arai, Remote sensing satellite image database system allowing image portion retrievals utilizing principal component which consists spectral and spatial features extracted from imagery data, International Journal of Advanced Research in Artificial Intelligence, 2, 5, 32038, 2013.

\section{AUTHORS' PROFILE}

Kohei Arai, He received BS, MS and PhD degrees in 1972, 1974 and 1982, respectively. He was with The Institute for Industrial Science and Technology of the University of Tokyo from April 1974 to December 1978 also was with National Space Development Agency of Japan from January, 1979 to March, 1990. During from 1985 to 1987, he was with Canada Centre for Remote Sensing as a Post Doctoral Fellow of National Science and Engineering Research Council of Canada. He moved to Saga University as a Professor in Department of Information Science on April 1990. He was a councilor for the Aeronautics and Space related to the Technology Committee of the Ministry of Science and Technology during from 1998 to 2000. He was a councilor of Saga University for 2002 and 2003. He also was an executive councilor for the Remote Sensing Society of Japan for 2003 to 2005. He is a Science Council of Japan Special Member since 2012. He is an Adjunct Professor of University of Arizona, USA since 1998. He also is Vice Chairman of the Science Commission "A" of ICSU/COSPAR since 2008 then he is now award committee member of ICSU/COSPAR. He wrote 55 books and published 620 journal papers as well as 450 conference papers. He received 66 of awards including ICSU/COSPAR Vikram Sarabhai Medal in 2016, and Science award of Ministry of Mister of Education of Japan in 2015. He is now Editor-in-Chief of IJACSA and IJISA. http://teagis.ip.is.sagau.ac.jp/index.html. 